\title{
س
}

$>\mathrm{DE}$

$\stackrel{1=1}{\simeq}$ PÉDAGOGIE

\author{
Recherches en éducation
}

158 | janvier-mars 2007

Varia

\section{BERNSTEIN Basil. Pédagogie, contrôle symbolique et identité : théorie, recherche, critique}

traduit par Ginette Ramognino-Le Déroff \& Philippe Vitale. Sainte-Foy [Québec] : Presses de l'université Laval, 2007. - 316 p. (Sociologie contemporaine)

\section{Sophia Stavrou}

\section{OpenEdition}

\section{Journals}

Édition électronique

URL : http://journals.openedition.org/rfp/532

DOI : $10.4000 /$ rfp.532

ISSN : 2105-2913

Éditeur

ENS Éditions

Édition imprimée

Date de publication : 1 mars 2007

Pagination : 165-168

ISBN : 978-2-7342-1080-1

ISSN : 0556-7807

\section{Référence électronique}

Sophia Stavrou, « BERNSTEIn Basil. Pédagogie, contrôle symbolique et identité : théorie, recherche, critique », Revue française de pédagogie [En ligne], 158 | janvier-mars 2007, mis en ligne le 23 septembre 2010, consulté le 22 septembre 2020. URL : http://journals.openedition.org/rfp/532 ; DOI : https://doi.org/ $10.4000 /$ rfp.532

Ce document a été généré automatiquement le 22 septembre 2020.

(c) tous droits réservés 


\section{BERNSTEIN Basil. Pédagogie, contrôle symbolique et identité : théorie, recherche, critique}

traduit par Ginette Ramognino-Le Déroff \& Philippe Vitale. Sainte-Foy [Québec] : Presses de l'université Laval, 2007. - 316 p. (Sociologie contemporaine)

\section{Sophia Stavrou}

\section{RÉFÉRENCE}

BERNSTEIN Basil. Pédagogie, contrôle symbolique et identité : théorie, recherche, critique / traduit par Ginette Ramognino-Le Déroff \& Philippe Vitale. Sainte-Foy [Québec] : Presses de l'université Laval, 2007. - 316 p. (Sociologie contemporaine

Dans cet ouvrage-testament, Basil Bernstein met en lumière l'aboutissement de l'œuvre d'une vie, d'un questionnement dynamique qui ne cesse d'être révisé et réactualisé sur le thème si permanent des inégalités sociales en éducation. Une théorisation qui ne se contente pas d'expliquer les inégalités scolaires, mais qui propose un important modèle de description du processus de transmission et d'acquisition. Une analyse qui se veut sociologique par son souci de dévoiler le passage du niveau macro-social (les rapports sociaux, les relations de pouvoir et de contrôle) au niveau micro-social, (la structuration des curricula, la pédagogie, la formation de la conscience).

2 En dépit de son absence de la scène intellectuelle française depuis les années 1970 Langage et classes sociales étant son seul ouvrage jamais traduit en français ${ }^{1}-$, son œuvre, appréciée au niveau international, est aujourd'hui plus que jamais d'actualité dans le contexte des politiques éducatives européennes et nationales en matière de réformes curriculaires. La professionnalisation de l'enseignement, la pluridisciplinarisation des formations dans le supérieur constituent, entre autres, des terrains concrets de mise en œuvre de la conceptualisation bernsteinienne, celle d'une 
interrogation persistante sur les relations entre et au sein des discours pédagogiques, des pratiques, des savoirs, des groupes sociaux et sur les transformations de celles-ci.

3 L'ouvrage oscille entre élaboration conceptuelle complexe et analyse des problèmes du système éducatif. La première partie est consacrée à la construction complexe et minutieuse d'un modèle de description de ce qu'on peut appeler le «relais» des inégalités sociales dans l'éducation. Autrement dit, l'auteur se donne pour objectif de dévoiler la manière dont les inégalités sont « véhiculées " par le système éducatif en mettant l'accent sur son fonctionnement et ses caractéristiques internes : le processus de sélection et d'organisation des savoirs dans le discours et dans la pratique pédagogiques.

4 Par ailleurs, l'originalité et le caractère heuristique du projet bernsteinien résident dans cette tentative de renouvellement de l'approche traditionnelle des inégalités scolaires et notamment les théories de la reproduction culturelle dont il critique les insuffisances: "Ce qu'il est intéressant de constater c'est que la structure qui permet réellement au pouvoir d'être relayé, au pouvoir d'être transmis, n'est pas elle-même soumise à l'analyse. Paradoxalement, ce qui manque aux théories de la reproduction culturelle c'est l'analyse interne de la structure du discours [pédagogique] lui-même, or c'est la structure de ce discours, la logique de ce discours, qui fournissent les moyens par lesquels il peut véhiculer les relations externes de pouvoir» (p. 4). La plupart des théories de la reproduction se contentent d'analyser les relations de l'élève avec le «texte pédagogique " (le curriculum et la pédagogie légitimes et toute autre représentation légitime orale, visuelle, spatiale, posturale - d'attitude et de position) suivant sa classe sociale, son sexe et ses attributs sociaux. Ce sont là des relations extérieures à l'institution éducative qui pèsent sur la réussite scolaire du sujet. Or, Bernstein note que les inégalités scolaires ne sont pas directement liées aux inégalités sociales, mais, sont (re)produites à travers le système de transmission des savoirs. Cet intermédiaire est constitué par des règles de structure et de fonctionnement qui lui sont propres, lesquelles construisent le texte pédagogique et lui donnent sa forme et ses caractéristiques distinctives. En ce sens, ce sont les relations internes que suppose le système de transmission (entre savoirs à acquérir, entre les pratiques, entre individus, etc.) qui permettent de comprendre comment se fabrique l'inégalité scolaire. Ce sont celles-ci qu'il faut examiner.

5 La construction conceptuelle de Basil Bernstein peut et doit être perçue comme un cadre descriptif riche, destiné à être testé par les chantiers empiriques de la sociologie et des sciences de l'éducation. La classification et le cadrage qui sont les concepts transversaux de son œuvre ouvrent des perspectives intéressantes quant à l'étude des situations d'apprentissage, de la réalité des projets pédagogiques et institutionnels. Ces deux concepts traduisent respectivement le degré et la forme des relations de pouvoir et des relations de contrôle entre et au sein des savoirs, des discours et des pratiques, établies institutionnellement dans le processus de transmission. Citons quelques exemples de l'examen de ces relations. Comment les savoirs sont-ils catégorisés et classifiés au sein du programme d'enseignement? Quel est l'ordre de leur transmission? Comment ces savoirs sont-ils découpés les uns des autres dans l'apprentissage? Quel est le degré de contrôle des enseignants ou des élèves sur le processus de transmission et d'acquisition? L'approche bernsteinienne fournit le cadre analytique qui permet de penser les principes généraux de description de ces relations afin d'explorer les observations empiriques. Il s'agit, donc, de partir de ce cadre 
descriptif des relations de classification et de cadrage pour comprendre comment celles-ci sont construites et comment celles-ci produisent des formes d'inégalisation en éducation. L'ouvrage, résolument théorique, ne manque toutefois pas d'exemples de mise à l'épreuve empirique empruntés aux recherches effectuées par l'auteur et par différents chercheurs des quatre coins du monde, élèves de Bernstein.

Le premier chapitre reprend le concept de départ du sociologue, le concept des codes $d u$ savoir scolaire qui traduit cette idée de l'intériorisation de l'ordre social dans le contexte de la pratique pédagogique, au travers des formes d'enseignement. Selon l'auteur, les relations de pouvoir et de contrôle social qui s'exercent dans la société structurent, dans l'éducation, les principes de classification et de cadrage au sein des systèmes du curriculum, de la pédagogie et de l'évaluation. Il y a ici la tentative de saisir ce qui fait la légitimité d'un texte pédagogique aux yeux des transmetteurs et des récepteurs et la manière dont cette légitimité est construite à partir des enjeux macro-sociaux. De la même manière que le pouvoir structure légitimement les groupes sociaux dans la société, la classification crée des frontières entre les discours dans l'enseignement ; elle établit une certaine forme de division du travail cognitif. Et de même que le contrôle social établit des formes légitimes de communication pour chaque groupe dans l'espace social, le cadrage régule les relations sociales dans la pratique pédagogique; il maintient cette division du travail cognitif par le contrôle sur la sélection de ce qui va être transmis, sur la séquence et les critères de transmission et sur la structuration de la relation pédagogique. La manière dont la classification et le cadrage opèrent dans le "texte» fournit les principes qui structurent, respectivement, les règles de reconnaissance du contexte pédagogique (comment reconnaître la spécialisation du contexte de communication par rapport aux autres) et les règles de réalisation du processus de transmission et d'acquisition (comment produire de la communication qui soit considérée comme légitime dans ce contexte). Ces deux types de règles permettent, en retour, de mettre en lumière les caractéristiques du code pédagogique et ses fondements sociaux. Force est de constater que la loupe analytique n'est pas focalisée sur l'observation de la reproduction des relations de pouvoir et de contrôle dans l'enseignement, mais, sur la compréhension des principes généraux de classification et de cadrage par lesquels cette reproduction s'opère.

Le deuxième chapitre constitue un prolongement de ce modèle de description, prolongement qui apparaît comme une réponse aux critiques adressées aux concepts de code élaboré/code restreint de la part à la fois des sociologues qui ont tenté de les mettre à l'épreuve empirique et de Bernstein lui-même: "cette approche a pris [le discours] comme une donnée et n'a pas examiné sa forme [...] elle a été entièrement focalisée sur les pratiques, les contextes et les sens au niveau des interactions » (p. 207). Il fallait, donc, pour l'auteur revenir à la question du processus de construction du discours pédagogique par l'intermédiaire de ce qu'il a appelé le schème pédagogique et ses trois types de règles: les règles de distribution, les règles de recontextualisation et les règles d'évaluation. Avec le concept de recontextualisation Bernstein passe en effet à un autre niveau d'analyse, plus proche d'une sociologie de la connaissance. Le lecteur est amené à réfléchir sur l'évolution de la théorie : alors que le code permet de rendre compte des réalisations spécifiques du discours dans la pratique pédagogique, le principe de recontextualisation fournit les conditions de possibilité de ces réalisations ; c'est-à-dire, pourquoi et comment la structuration et le fonctionnement du système permettent une certaine sélection et une certaine organisation potentielles des savoirs qui seront mises en œuvre dans l'enseignement. La recontextualisation est un principe qui «s'approprie 
de manière sélective, qui relocalise, refocalise et relie des discours entre eux pour constituer son propre ordre » (p. 33) ; un principe qui introduit un discours d'instruction (des savoirs et des compétences) dans un discours de régulation (d'ordre social). Cet espace de transformation du texte pédagogique est considéré comme un espace de lutte entre groupes sociaux, entre agents de recontextualisation, pour la définition du «quoi » et $\mathrm{du}$ "comment» du discours pédagogique et où l'idéologie peut jouer un rôle fondamental. Il inclut aussi bien les champs officiel (agences de l'État et des autorités locales) et pédagogique que des champs extérieurs à l'éducation, comme le champ politique ou économique. Cette confrontation de différents agents de recontextualisation pour la construction du discours pédagogique révèle un aspect pluriel et dynamique du modèle qui neutralise ainsi dans l'analyse ce qui pourrait apparaître comme du déterminisme culturel.

C'est cette perspective des politiques de recontextualisation que développent les deux chapitres suivants, dans une dialectique entre construction conceptuelle et discussion sur les transformations actuelles du système éducatif. La typologie des formes curriculaires et pédagogiques d'enseignement qu'établit Bernstein vise à mettre l'accent sur les enjeux socioculturels que présente la recontextualisation des savoirs dans l'objectif de la formation d'une identité pédagogique. Le passage d'un modèle de " compétence " à un modèle de "performance » est illustré à travers l'exemple de l'Angleterre sous le régime Thatcher : à la fois une décentralisation de la gestion des institutions locales et une centralisation du contrôle et du financement. Ce modèle dévoile la manière dont les rapports externes peuvent structurer le contenu de l'éducation. Dans ce cas de figure, c'est le processus de recontextualisation (et plus précisément les agents qui y participent) qui incite à l'introduction d'une culture pédagogique dans une culture de compétition entrepreneuriale et qui produit des transformations à l'intérieur même du texte pédagogique. Le changement s'opère dans l'identité pédagogique à construire : de l'introjection de la connaissance (centrée sur le contrôle social qui s'exerce sur la formation de la conscience) à la projection (centrée sur la valeur instrumentale du produit dans l'économie). En ce sens, les réformes actuelles de l'enseignement qui se veulent vecteurs de l'ouverture des frontières entre champs $\mathrm{du}$ savoir (savoir disciplinaire/transversal, savoir scientifique/pratique ou quotidien, etc.) ne peuvent en aucun cas être considérées comme abstraites des relations de pouvoir et de contrôle social qui structurent le système de transmission et d'acquisition. Nous sommes peut-être arrivés, souligne l'auteur, à dépasser les clôtures produites par les identités des disciplines, orientées vers leur propre développement de par les frontières rigides et les hiérarchies qu'elles maintiennent entre elles. Mais, le processus de transmission des savoirs s'inscrit toujours dans des clôtures, des relations de classification et de cadrage structurées par les enjeux socioculturels, politiques et, désormais, économiques de la société. En d'autres termes, la stratification des identités, des institutions, des savoirs et des pratiques ne disparait pas ; elle prend une nouvelle forme, produite par un nouveau principe de recontextualisation; des éléments qui alimentent le débat actuel sur les réformes de l'enseignement supérieur. Les préoccupations de l'auteur sont également reflétées au travers du phénomène observé du divorce entre "le connaisseur" et "la connaissance" (chapitre 5). Tandis que l'université médiévale a établi une relation entre l'ésotérique et l'exotérique dans le rapport du sujet à la connaissance aujourd'hui les deux se séparent: deux marchés coexistent, celui de la connaissance (et de son économie) et celui des producteurs et des usagers potentiels des connaissances. Un point intéressant pour réorienter le débat: 
aujourd'hui on se soucie plus de l'utilité et de l'efficacité de la connaissance dans le domaine de la pratique que du statut de la connaissance elle-même et de son apport dans le développement de l'esprit, dans le rapport du sujet à cette connaissance. Si l'étudiant est réduit à quelqu'un qui « applique » des savoirs, aurait-on alors abandonné le rôle d'instruction de l'institution éducative ? Et quelles seraient les conséquences de ce nouveau rapport « exotérique » de l'individu à la connaissance?

Bernstein revient dans la deuxième partie de son ouvrage sur des questions épistémologiques, à la fois pour mettre en lumière les conditions d'élaboration de sa propre théorie (en relation permanente avec la recherche qu'il a menée lui-même et avec celle des doctorants qu'il a dirigée), mais, aussi, en plaidant pour un langage de description élaboré dans un va-et-vient entre conceptualisation et confrontation empirique. Dans l'approche proposée, ne s'inscrivant ni dans une démarche hypothético-déductive ni dans une démarche inductive, ce sont les principes de description que le chercheur doit pouvoir repérer; des principes de description qui permettent, d'une part, de définir et de rendre compte des « relations empiriques » à étudier et, d'autre part, de reformuler le langage conceptuel. C'est à travers cette même approche que Bernstein établit les principes de description du passage des relations de distribution du pouvoir et de contrôle social à la structuration de la communication par l'intermédiaire de la classification et du cadrage du "texte pédagogique »; un langage de description qui, malgré sa complexité et son haut degré d'abstraction, peut dépasser le contexte de l'institution éducative et expliquer d'autres cas de relations de transmission au sein des pratiques d'interaction (relation familiale, médicale, etc.). Ce qui fait la richesse de l'approche bernsteinienne n'est pas uniquement la tentative de remonter au général pour expliquer le particulier; c'est également cette réflexivité portée sur la relation entre théorie et recherche qui conduit son modèle de description à un développement perpétuel ouvert à tous les chercheurs.

10 C'est, par ailleurs, de cette perspective de développement et de révision du modèle que témoignent les quatre derniers chapitres de cet ouvrage. À partir des critiques adressées à la thèse des "codes sociolinguistiques" (élaborée dans ses premiers travaux), l'auteur retourne aux fondements du concept pour appuyer son idée d'une base sociale qui structure les modalités de communication, leurs principes de distribution et leurs effets différentiels. La spécificité de son approche repose sur la distinction entre la mise en évidence des effets différentiels de l'apprentissage et la compréhension du processus qui produit ces effets. Bernstein s'inscrivant dans cette dernière perspective trace son propre chemin théorique par rapport aux autres sociologues de la reproduction et notamment par rapport à Pierre Bourdieu (chapitres 8 et 10). Comme il explique lui-même, "j'ai essayé de dépasser les critiques particulières pour les utiliser comme moyens d'éclaircissement, et parfois comme moyens de développement, de mon texte initial » (p. XVIII). Ce développement, qui, en grande partie, lui vaut l'arrivée de son œuvre en France, est précisément l'accent mis sur les within relations, c'est-à-dire l'analyse des relations à l'intérieur de l'institution éducative, du discours pédagogique, qui permettent d'expliquer les effets de l'apprentissage à partir des enjeux macrosociaux. La place dominante, dans la théorie, donnée au « discours » au détriment du « code » (chapitre 9) refocalise le débat scientifique sur les propriétés internes du texte pédagogique et, par conséquent, sur le contexte social dans lequel celui-ci est construit.

11 Cet ouvrage fera sans doute date pour avoir institué une sociologie descriptive, relationnelle et pragmatique de l'école, qui explique les inégalités scolaires en les 
dépassant. Une approche qui vise une question de tous les temps et de tous les contextes: celle du processus de transmission et d'acquisition, qui se réclame d'une sociologie de la reproduction sans s'inscrire dans une perspective bourdieusienne. C'est là l'intérêt de l'approche bernsteinienne : ce qu'il importe de comprendre n'est pas ce qui est reproduit, mais, comment cela est reproduit. Sans pour autant tomber dans un relativisme scientifique et culturel, Bernstein établit comme unité d'analyse le processus éducatif et pédagogique et, plus précisément, les formes de relation qu'il inclut qui permettent ou qui ne permettent pas de relayer les rapports macro-sociaux dans l'école. En définitive, il s'agit d'un modèle de description des conditions de (non-)possibilité des inégalités scolaires. L'optimisme de l'auteur étant esquissé dans ce qui devrait constituer les «pages noires" de la reproduction sociale, le chantier du potentiel du changement s'offre à tous ceux qui s'intéressent à la question.

\section{NOTES}

1. Basil Bernstein, Langage et classes sociales: codes socio-linguistiques et contrôle social / traduit par Jean-Claude Chamboredon. Paris : Éd. de Minuit, 1975.

\section{AUTEURS}

SOPHIA STAVROU

Université de Provence, Laboratoire méditerranéen de sociologie (LAMES) 